\title{
Controlling Topological States in Topological/Normal Insulator Heterostructures
}

\author{
Marcio Costa, ${ }^{*},+\ddagger \odot$ Antônio T. Costa, ${ }^{\dagger, \S \odot ~ W a l t e r ~ A . ~ F r e i t a s, ~}{ }^{\|}$Tome M. Schmidt, \\ Marco Buongiorno Nardelli, ${ }^{\perp, \#}$ and Adalberto Fazzio ${ }^{*}, \dagger$ \\ ${ }^{\dagger}$ Brazilian Nanotechnology National Laboratory (LNNano), CNPEM, 13083-970 Campinas, Brazil \\ ${ }^{\ddagger}$ Centro de Ciências Naturais e Humanas, Universidade Federal do ABC, 09210-580 Santo André, São Paulo, Brazil \\ ${ }^{\S}$ Departamento de Física, Universidade Federal Fluminense, 24210-271 Niterói, Rio de Janeiro, Brazil \\ "Instituto de Física, Universidade Federal de Uberlândia, CP 593, 38400-902 Uberlândia, Minas Gerais, Brazil \\ ${ }^{\perp}$ Department of Physics and Department of Chemistry, University of North Texas, Denton, Texas 76203, United States \\ ${ }^{\#}$ Center for Materials Genomics, Duke University, Durham, North Carolina 27708, United States
}

\section{Supporting Information}

ABSTRACT: We have performed a systematic investigation of the nature of the nontrivial interface states in topological/normal insulator (TI/NI) heterostructures. On the basis of first principles and a recently developed scheme to construct ab initio effective Hamiltonian matrices from density functional theory calculations, we studied systems of realistic sizes with high accuracy and control over the relevant parameters such as TI and NI band alignment, NI gap, and spin-orbit coupling strength. Our results for IV-VI compounds show the interface gap tunability by appropriately controlling the NI thickness, which can be explored for device design. Also, we verified the preservation of an in-plane spin texture in the interface-gaped topological states.
Normal Insulator Thickness
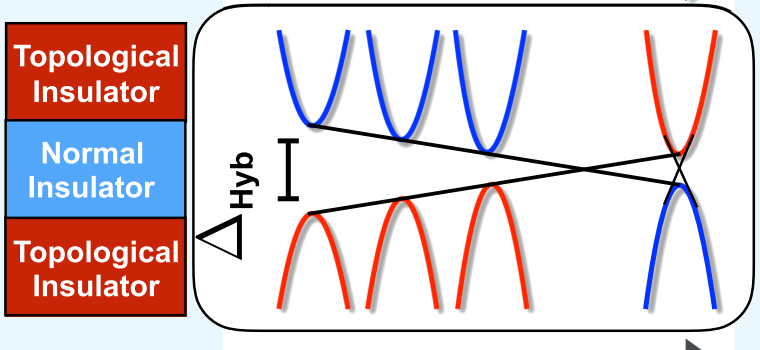

Normal Insulator Gap

\section{INTRODUCTION}

In 1970, Esaki and Tsu published a seminal paper in which they proposed a solid-state artificial structure, the superlattice (SL). ${ }^{1}$ This material is engineered as a periodic modulation of the composition of an alloy, with a period much larger than the host material lattice parameter (see Figure 1a). This new class of synthetic semiconductor structures allowed a profusion of new technological applications to appear. ${ }^{2}$ Nowadays, SLs are not restricted to the realm of semiconductors but are ubiquitous throughout solid-state physics. Such heterostructures are well controlled experimentally, where different techniques are used in their synthesis, e.g., molecular beam epitaxy and metalorganic vapor phase epitaxy deposition. Furthermore, SLs are fabricated from a wide range of materials, such as magnetic and nonmagnetic metals, insulators, and superconductors. ${ }^{3,4}$

More recently, topological insulators (TIs) ${ }^{5-7}$ were added to the previous list. ${ }^{8,9}$ TIs form a class of materials that present an insulating bulk with robust conducting states on their boundary. A question that arises naturally is how the TI properties are affected by interfacing with trivial materials, in a sequential way forming a SL. It is well known that on a TI surface the metallic topological states are Dirac-like with a spin texture that gives rise to a spin current protected from backscattering by time-reversal symmetry. Nevertheless, TIs interfaced with a regular or normal insulator (NI) are poorly understood. Some recent studies show unequivocally the appearance of Dirac cones at the TI/NI interface. ${ }^{10-18}$ Existing calculations $^{19-21}$ and recent experiments ${ }^{22}$ predict that states at the interface, located predominantly within the normal material, could acquire spin texture because of their proximity to the topological interface states. Now, let us consider a system composed of layers of TI and NI stacked in an alternating fashion, forming a one-dimensional superlattice. Such a system can bring novel technological applications, especially considering the sequence of interfacial nontrivial states and their spin orientation.

Our findings show a direct relationship between the normal insulator thickness $\left(L_{\mathrm{NI}}\right)$ and the appearance of topologically protected Dirac states (TPDS) at the SL interfaces. For a $L_{\mathrm{NI}}$ smaller than the critical thickness $\left(L_{\mathrm{C}}\right)$, the band structure will have the features of Figure $1 \mathrm{~b}$, with a hybridization gap $\left(\Delta_{\text {hyb }}\right)$ that is the result of TPDS interaction of adjacent interfaces. The linear dispersion Dirac states shown in Figure 1c will be present only for large $L_{\mathrm{NI}}$ (greater than $L_{\mathrm{C}}$ ). On the other hand, a metallic Dirac crossing is always present at the TI in

Received: July 31,2018

Accepted: September 28, 2018

Published: November 26, 2018 


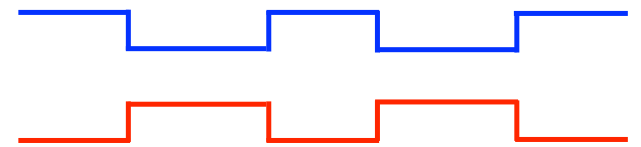

b

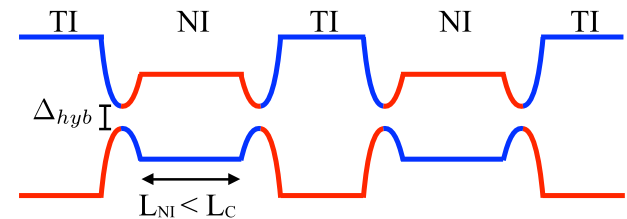

c

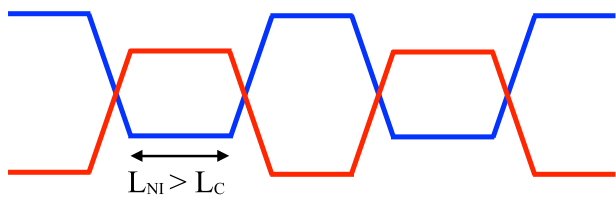

Figure 1. Schematic band structure for different heterostructures. (a) Regular semiconductor heterostructure. (b) Topological/normal insulator heterostructure $(\mathrm{TI} / \mathrm{NI})$ for NI thickness $\left(L_{\mathrm{NI}}\right)$ smaller than the critical value $\left(L_{\mathrm{C}}\right)$. The topologically protected Dirac states (TPDS) are strongly hybridizing originating the $\Delta_{\text {hyb }}$ gap. (c) TI/NI heterostructure where $L_{\mathrm{NI}}>L_{\mathrm{C}}$ and the TPBS are decoupled.

contact with the vacuum (surface), independent of the inner $L_{\mathrm{NI}}$ width. Also, our calculations point out an inverse relationship between the NI gap and the critical thickness $L_{C}$.

The paper is organized as follows: in Section 2, we give a brief description of the methodology used to construct the effective Hamiltonians and how the spin-orbit coupling (SOC) is included; we also compare these results to fully relativistic density functional theory (DFT) calculations for validation. The computational details are described in Section 3. In Section 4, the effective Hamiltonian results are discussed and compared to DFT calculations.

\section{METHODOLOGY}

This section briefly describes the methodology used to construct the effective Hamiltonians and the inclusion of the spin-orbit coupling (SOC) effect. The central idea is to map the plane waves (PWs) Hilbert space, in general composed of several thousand of PWs, onto the compact subspace spanned by the pseudoatomic orbitals (PAOs) $\left|\phi_{\mu}\right\rangle$ used to construct the atomic pseudopotentials. The Hamiltonians are constructed from the Kohn-Sham (KS) Bloch states $\left(\left|\Psi^{\mathrm{KS}}\right\rangle\right)$ projection onto the PAO basis set localized at the atomic sites. Considering the PAO basis set incompleteness, some KS states are not accurately described. These manifest themselves as unphysical eigenvalues and hybridizations. The solution is to apply a filtering procedure to shift these states outside the energy window of interest. The filtering procedure is based on the so-called projectability number $\left(p_{n}\right)$, which is defined as $p_{n}$ $=\left\langle\Psi_{n}|\hat{P}| \Psi_{n}\right\rangle$ and the operator $\hat{P}$ projects the KS states onto the PAO basis set. The interpretation of $p_{n}$ is straightforward; KS states with $p_{n} \approx 1(\approx 0)$ represent states that are well (poor) described by the given PAO basis set. In this work, the $p_{n}$ threshold is set to 0.95. This methodology has been successfully applied to different materials to compute many properties; for a full description, see refs 23-27. Since the PAO Hamiltonian resembles the traditional tight-binding (TB) Hamiltonians, we will refer to it as TB Hamiltonian.

Spin-orbit coupling effect is essential to describe the topological quantum states in TI. In our calculations, the SOC is introduced in two different ways: standard fully relativistic self-consistent DFT calculations ${ }^{28}$ and an effective approximation in the TB Hamiltonian. ${ }^{27,29}$ The effective SOC included in the TB Hamiltonian can be written as

$$
H_{\text {SOC }}=\lambda \mathbf{L} \cdot \mathbf{S}
$$

where $\lambda$ is the, orbital- and element-dependent, spin-orbit coupling strength, and $\mathbf{L}$ and $\mathbf{S}$ are the orbital and spin angular momentum operators, respectively. Figure 2 shows a
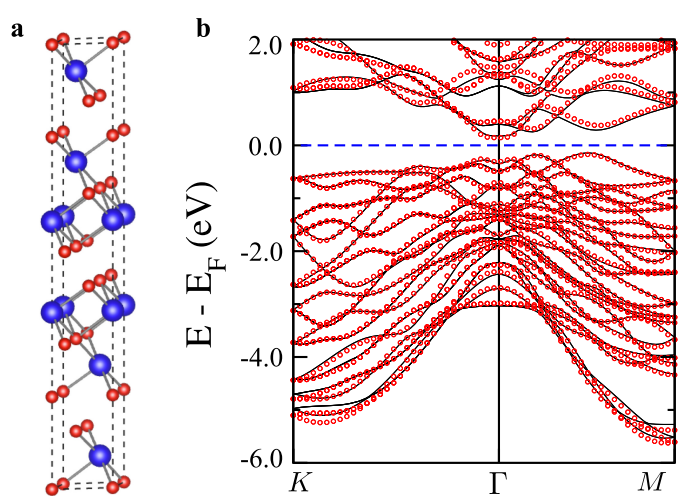

Figure 2. (a) $\mathrm{Bi}_{2} \mathrm{Se}_{3}$ bulk hexagonal crystal structure and its corresponding band structure. (b) $(K-\Gamma-M)$ for a fully relativistic DFT calculation (solid black line) compared to the TB + SOC (open red circles). The blue dashed line represents the Fermi level.

comparison between DFT with SOC (solid black line) and the $\mathrm{TB}+\mathrm{SOC}$ (open red circles) in $\mathrm{Bi}_{2} \mathrm{Se}_{3}$ hexagonal bulk band structure. The $\mathrm{Bi}$ and Se p-orbitals' SOC parameters used are $\lambda_{\mathrm{Bi}}=1.475 \mathrm{eV}$ and $\lambda_{\mathrm{Se}}=0.265 \mathrm{eV}$. These values are in good agreement with the literature ${ }^{30}$ and were obtained via a fitting procedure of the $\mathrm{Bi}$ and Se body-centered cubic DFT-SOC band structure. The agreement between the DFT and TB is excellent. Same degree of agreement is observed for a $\mathrm{Bi}_{2} \mathrm{Se}_{3}$ surface with four quintuple-layers (Qls), see Figure $3 a, b$, and in TI/NI heterostructures: to this end, we considered a heterostructure composed of two NI Qls between four TI Qls; see the inset in Figure 3c. In the TB Hamiltonian, such a heterostructure is constructed by setting $\mathrm{Bi}$ and $\mathrm{Se} S O C$ parameters to zero in the NI region, i.e., $\lambda_{\mathrm{Bi}}=\lambda_{\mathrm{Se}}=0$. The DFT calculation was performed with a $\mathrm{Bi}$ and Se nonrelativistic pseudopotential in the NI region to mimic the SOC absence. The DFT with SOC and TB + SOC heterostructure band structure are shown in Figure $3 \mathrm{c}$,d, respectively. In the bulk and surface cases, the agreement is outstanding. The bulk TB Hamiltonian is available in the Supporting Information along with the $\mathrm{Bi}_{2} \mathrm{Se}_{3}$ crystal unit cell.

The ideas described above are used to construct the heterostructures calculated in this work. From the Hamiltonian matrix of the $(1 \times 1 \times 1) \mathrm{Bi}_{2} \mathrm{Se}_{3}$ hexagonal supercell (details of the supercell are given in the next section), we can construct massive bulk supercells, up to $400 \AA$ in thickness $(1 \times 1 \times 16)$, with and without surfaces. These massive supercells preserve the exceptional agreement demonstrated previously.

\section{COMPUTATIONAL DETAILS}

We performed density functional theory (DFT) $)^{31,32}$ calculations using the plane wave package Quantum Espresso ${ }^{33,34}$ and Vienna ab initio simulation package code. ${ }^{35,36}$ The generalized gradient approximation ${ }^{37}$ was employed to treat the correlation among electrons with the Perdew-Burke- 

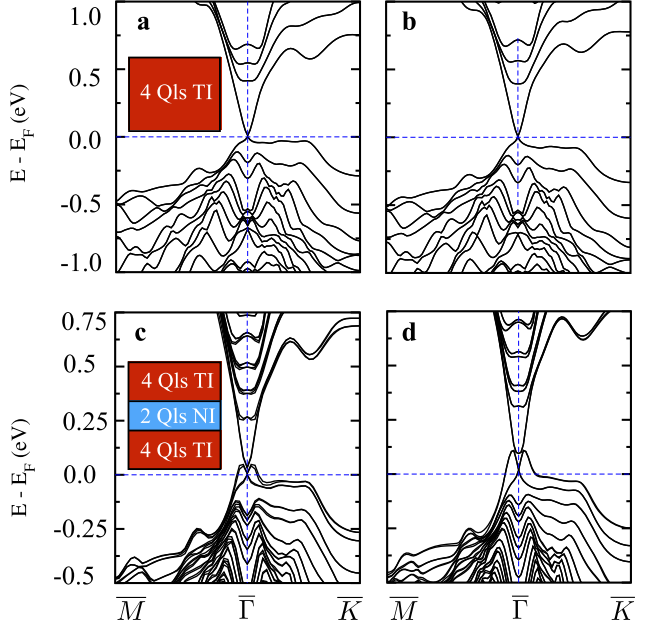

Figure 3. Comparison between the DFT-SOC and TB + SOC band structures. $\mathrm{Bi}_{2} \mathrm{Se}_{3}$ four-Qls surface band structure (a) DFT-SOC and (b) $\mathrm{TB}+$ SOC. A finite heterostructure with two Qls of $\mathrm{Bi}_{2} \mathrm{Se}_{3}$ without SOC, normal insulator (NI), between two four- $\mathrm{Qls}$ of $\mathrm{Bi}_{2} \mathrm{Se}_{3}$ with SOC, topological insulator (TI), (c) DFT-SOC, and (d) TB + SOC. The inset of panel (c) shows the schematic representation of the TI/NI/TI heterostructure. The red (blue) regions represent the TI (NI) layers.

Ernzerhof parametrization. ${ }^{37}$ The Bi and Se ionic potentials are described using projector augmented wave pseudopotentials ${ }^{38}$ with $\mathrm{s}, \mathrm{p}$, and $\mathrm{d}$ as valences. The $\mathrm{Bi}_{2} \mathrm{Se}_{3}$ is a rhombohedral crystal with space group $D_{3 d}^{5}(R \overline{3} m)$ and a five-atoms unit cell. To facilitate the interface construction and results' interpretation, a $\mathrm{Bi}_{2} \mathrm{Se}_{3}$ hexagonal cell composed of 15 atoms was used; see Figure 2a. This cell contains three Qls where the intra (inter) Ql chemical bonding is of ionic/covalent (van der Waals) character. The experimental lattice parameter is adopted with $a=4.134 \AA$ and $c=28.63 \AA$. The reciprocal space sampling uses the Monkhorst-Pack scheme with $k$ meshes of $19 \times 19 \times 3$ and $19 \times 19 \times 1$ for bulk and surface calculations, respectively. We also performed a fully relativistic calculation for a heterostructure of $\mathrm{Bi}_{2} \mathrm{Se}_{3}$ and a real trivial system $\mathrm{Sb}_{2} \mathrm{Se}_{3}$. Since both systems present similar lattice parameters, we used the average lattice parameter for the hexagonal plane $(a=4.107 \AA)$ and the vertical $c=30.90 \AA$ for $\mathrm{Sb}_{2} \mathrm{Se}_{3}$. At the interface, the average of both structures was used, $c=29.765 \AA$. The calculated topological invariant using these parameters gives $Z_{2}=1$ for $\mathrm{Bi}_{2} \mathrm{Se}_{3}$ and $Z_{2}=0$ for $\mathrm{Sb}_{2} \mathrm{Se}_{3}$.

\section{RESULTS AND DISCUSSION}

4.1. Bulk TI/NI Superlattices. Using the TB method, where the NI region is described by suppressing the SOC, in this section we construct an infinite periodically bulk TI/NI superlattice (without any surface). In Figure 4, the $\mathrm{Bi}_{2} \mathrm{Se}_{3}$ bulk band structure is shown (a) without SOC and (b) with SOC; the SOC increases the gap from $0.16 \mathrm{eV}$ (direct gap) to 0.33 $\mathrm{eV}$ (indirect gap), which is in agreement with previous calculations. ${ }^{30}$ In panel (c), we show the band structure for a $4 / 8$ (TI/NI) infinite heterostructure where the topological states at the interface become massive and a hybridization gap $\left(\Delta_{\text {hyb }}\right)$ of $42 \mathrm{meV}$ appears at the $\bar{\Gamma}$ point. The $\Delta_{\text {hyb }}$ gap originates from the TPDS hybridization through the NI material. Its value is reduced by increasing the NI thickness, and the TPDS re-emerge above a critical thickness $\left(L_{\mathrm{C}}\right)$ around $200 \AA$ ( $\approx 21 \mathrm{Qls})$; see panel (d). These superlattices

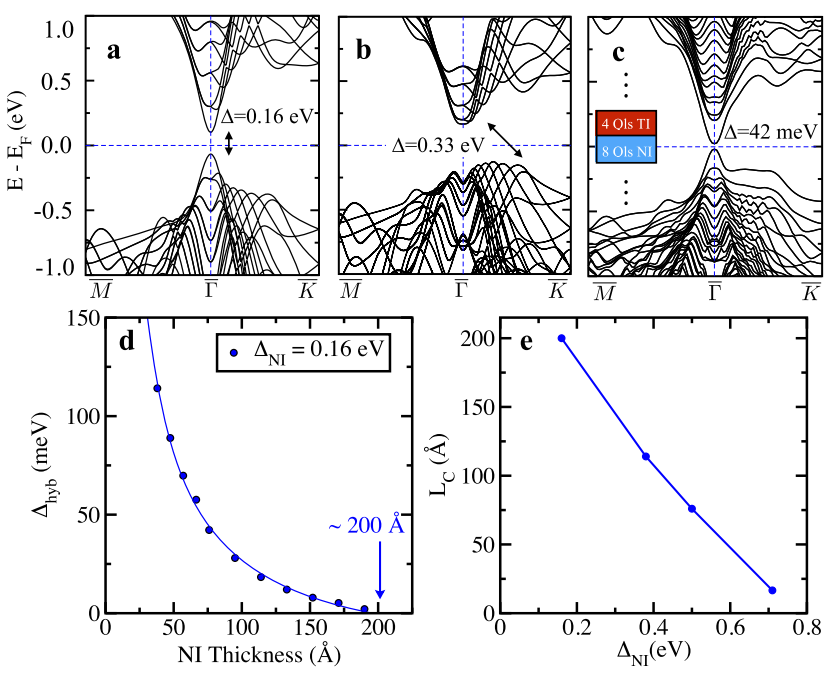

Figure 4. $\mathrm{Bi}_{2} \mathrm{Se}_{3}$ bulk band structure (a) without and (b) with SOC. (c) $4 / 8$ (TI/NI) heterostructure band structure; a $42 \mathrm{meV}$ hybridization gap $\left(\Delta_{\text {hyb }}\right)$ is obtained. (d) $\Delta_{\text {hyb }}$ evolution as the NI thickness is increased. (e) Relation between the NI gap $\left(\Delta_{\mathrm{Nl}}\right)$ and the critical thickness $\left(L_{\mathrm{C}}\right)$ is shown.

are chemically and structurally perfect; as a consequence, there is no Rashba splitting, which would appear because of inversion symmetry breaking, as reported in ref 39 . In a real heterostructure, the NI region is formed by different materials; thus, it is important to understand the relationship between the NI gap $\left(\Delta_{\mathrm{NI}}\right)$ and $L_{\mathrm{C}}$. To simulate a variation on the $\Delta_{\mathrm{NI}}$ gap within the TB model, a local potential is applied to $\mathrm{Bi}$ and $\mathrm{Se}$ p-states. As shown in Figure 4e, there is an inverse relationship between $\Delta_{\mathrm{NI}}$ and $L_{\mathrm{C}}$. By increasing $\Delta_{\mathrm{NI}}$ to $0.5 \mathrm{eV}$, the $L_{\mathrm{C}}$ is reduced to $75 \AA(\approx 8 \mathrm{Qls})$. This finding is important to guide the experimental search for suitable constituent materials of heterostructures with desired properties.

4.2. $\mathrm{TI} / \mathrm{NI}$ Heterostructure Terminated in $\mathrm{TI}$ or NI. In this section, we investigate heterostructures with vacuum terminations. Two different constructions will be explored: one NI between two TI regions (TI/NI/TI) and one TI between two NI (NI/TI/NI) regions as building blocks for realistic TI/ NI superlattices. In Figure 5, we show the band structure for the $4 / 8 / 4$ (TI/NI/TI) sandwich, projected on the interfaces. The plot shows states with high (above 60\%) spectral weight at the indicated regions (the insets of panels (a) and (b)). In Figure $5 \mathrm{a}, \mathrm{b}$, the NI region gap is $0.16 \mathrm{eV}$ (no SOC potential is applied and $\lambda_{\mathrm{Bi}}=\lambda_{\mathrm{Se}}=0.0$ ), and the TI and NI bands are perfectly aligned, as indicated in panel (c). The TPDS are observed at the surface, and their dispersions are consistent with the four-Qls $\mathrm{Bi}_{2} \mathrm{Se}_{3}$ surface. However, the TPDS at the $\mathrm{TI} / \mathrm{NI}$ interface are replaced by states with parabolic dispersions and a $42 \mathrm{meV}$ hybridization gap. This is in perfect agreement with the situation in the $4 / 8$ infinite superlattice, including the size of the hybridization gap. In panels (d) and (e), we investigate the effect of increasing the NI band gap on the TPDS. For a gap of $0.5 \mathrm{eV}$, no significant change is observed at the surface. At the NI interfaces, however, the TPDS are restored, with an increased dispersion and an upward energy shift. This result strongly suggests that large NI band gaps effectively decouple the two TI/NI interfaces. Similar to that in ref 40, we explore the relation between band alignment and the TPDS formation. As in the previous cases, the surface TPDS are not significantly affected by the band 


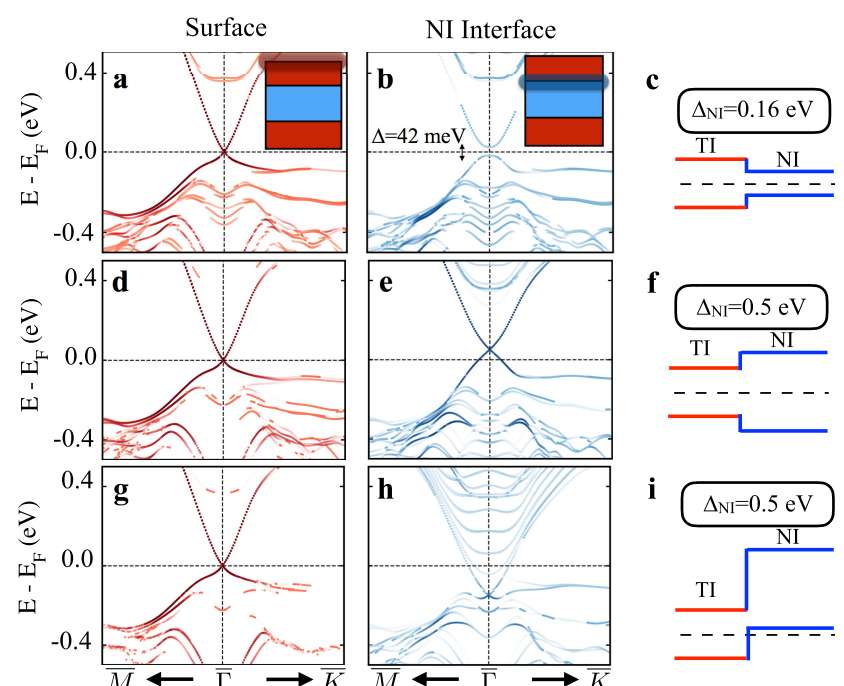

Figure 5. Spatial projected band structure for a $4 / 8 / 4$ (TI/NI/TI) finite heterostructure. Projection onto the outermost two TI Qls at the surface: (a) $\Delta_{\mathrm{NI}}=0.16 \mathrm{eV}$ (bands aligned), (d) $\Delta_{\mathrm{NI}}=0.50 \mathrm{eV}$ (bands aligned), and (g) $\Delta_{\mathrm{NI}}=0.50 \mathrm{eV}$ (bands not aligned). In (b), (e), and (h), the projection is calculated at $\mathrm{TI} / \mathrm{NI}$ interface for the respective heterostructures. The shaded areas of the insets of panels (a) and (b) show the projected regions. In (c), (f), and (i), a schematic representation of the band alignment for the respective heterostructure is shown.

misalignment (panel $(\mathrm{g})$ ), while the ones at the NI/TI interface are dramatically shifted downward in energy (panel (h)).

The next heterostructure is composed of a TI between two $\mathrm{NI}$ (NI/TI/NI) with equal thicknesses. With such construction, the dispersion of TPDS acquires a parabolic shape, as seen in Figure 6a. This larger dispersion results in the reduction of the Fermi velocity when compared with the fourQls surface linear dispersion; see Figure 3b. Another important feature is the localization, in energy, of the bulk states between $\bar{\Gamma}$ and $\bar{M}$. For the four-Qls surface, this state is located $90 \mathrm{meV}$
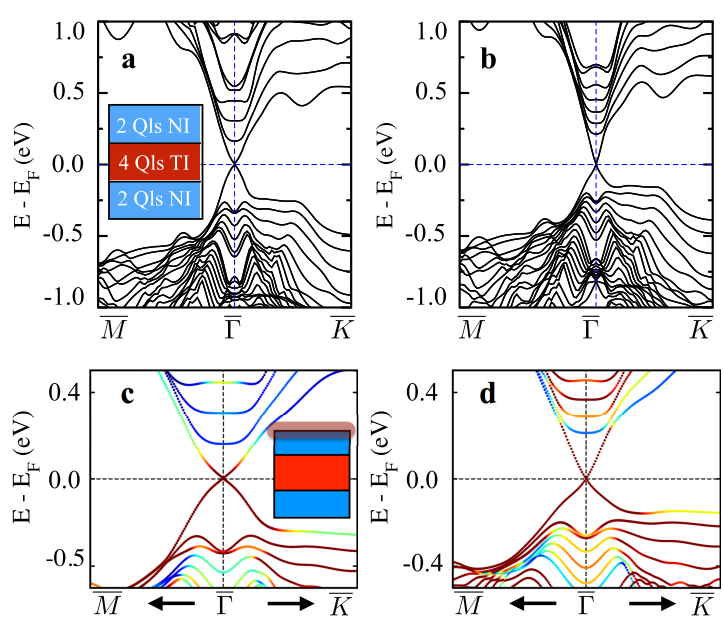

Figure 6. Band structure for a $2 / 4 / 2$ (NI/TI/NI) finite heterostructure. (a) $\lambda_{\mathrm{Bi}}=0.0 \mathrm{eV}$ and (b) $\lambda_{\mathrm{Bi}}=1.10 \mathrm{eV}$. Eigenvalues spatial projection: (c) $\lambda_{\mathrm{Bi}}=0.0 \mathrm{eV}$ and (d) $\lambda_{\mathrm{Bi}}=1.10 \mathrm{eV}$. Dark red (dark blue) represents high (low) spectral weight at the projected regions. The inset of panel (c), shaded region, highlights the projected region. below the Dirac point (DP). Once the TI is placed in between two NI layers, these states shift down to $240 \mathrm{meV}$ below the DP. This effect is due to the absence of SOC at the surface since $\lambda_{\mathrm{Bi}}=\lambda_{\mathrm{Se}}=0.0$. As an exercise to understand this behavior, the Se SOC is restored $\left(\lambda_{\mathrm{Se}}=0.265 \mathrm{eV}\right)$ and the Bi SOC is increased to $1.10 \mathrm{eV}$ in the NI region. The bulk states are 150 $\mathrm{meV}$ below DP and move toward the surface value as $\lambda_{\mathrm{Bi}} \rightarrow$ $100 \%$. This energy shift contributes to the reduction of the influence of these bulk states on the $\mathrm{Bi}_{2} \mathrm{Se}_{3}$ transport properties. ${ }^{41,42}$ This has been observed for $\left(\mathrm{Bi}_{x} \mathrm{Sb}_{1-x}\right)_{2} \mathrm{Se}_{3}$ alloys, ${ }^{43}$ where $\mathrm{Sb}$ reduces the overall SOC. Moreover, panels (c) and (d) show the spatial localization of the TPDS; a significant penetration of these states in the NI region is observed. This can be exploited to establish contacts for transport measurements or attain functionalization, for example.

4.3. Multilayers of $\mathrm{TI} / \mathrm{NI}$ Superlattice Terminated in TI. We also investigate a superlattice composed of multilayers of topological and trivial insulators. The modulation is performed by controlling the spin-orbit coupling, similar to the control of topological phase by doping, as reported in ref 8 . At the TI surface, the TPDS are always present. Nevertheless, at the TI/NI interface, these states are absent because of a strong hybridization between subsequent interfaces. Figure 7, left panel, shows the schematic representation for a $4 / 2$ (TI/ $\mathrm{NI})$ heterostructure composed of $22 \mathrm{Qls}(\approx 200 \AA)$. In the TI region (red), full Bi and Se SOC are adopted. In the NI region (blue), the Se SOC is kept fixed at its full value and the Bi SOC is varied from 50 to $0 \%$ of its full value. The Dirac-like states come from the TI in the surface (red in panels $(\mathrm{a})-(\mathrm{c})$ ), and no contributions come from the internal TI region (see panels $(\mathrm{d})-(\mathrm{f}))$. We can understand this by thinking about the $4 / 2$ (TI/NI) structure as a unit cell of a "new" or "meta" material. This metamaterial is indeed a topological insulator with $Z_{2}=1$, since there is only one band inversion in the first Brillouin zone at the $\Gamma$ point. In this way, for such thin films of the TI/NI metamaterial, we predict surface metallic topological states and a gapped bulk, exactly as shown in Figure 7. Naturally, by increasing the NI region greater than the critical thickness, as shown above, gapless interface TPDS will be restored. It is important to note that the Dirac crossing at the interfaces will only succeed for a large NI region, when the topological states of adjacent interfaces are effectively decoupled from each other by the intervening NI layers.

4.4. $\mathrm{Bi}_{2} \mathrm{Se}_{3} / \mathrm{Sb}_{2} \mathrm{Se}_{3} / \mathrm{Bi}_{2} \mathrm{Se}_{3}$ Heterostructure. To complement our discussion above, fully relativistic first-principles calculations of the $\mathrm{Bi}_{2} \mathrm{Se}_{3}$ (TI) interfaced with $\mathrm{Sb}_{2} \mathrm{Se}_{3}$ (NI) were performed. Since both materials are lattice matched, the heterostructure preserves the bulk topological properties. For a periodic supercell composed of $4 / 2$ (TI/NI), a gap is formed (see Figure 8). There are topological surface bands at the interfaces that come mostly from the TI side; however, the global gap is preserved. By cutting our TI/NI supercell to produce top and bottom surfaces, a different picture is observed, confirming our TB results above. As shown in Figure 9, the TPDS re-emerge at the surface and a gap is present at the $\mathrm{Sb}_{2} \mathrm{Se}_{3}$ interface. The whole system behaves as a unique TI material, gapped inside but metallic at the surfaces. As shown in Figure 9g, the TPDS are Rashba-like states, due to the coupling between opposite interface TPBS, as observed for surface states in thin films. ${ }^{44}$ The asymmetric distributions of the top and bottom surface states (Figure 9f) arise because of a symmetry breaking induced by the NI material. This breaks the 

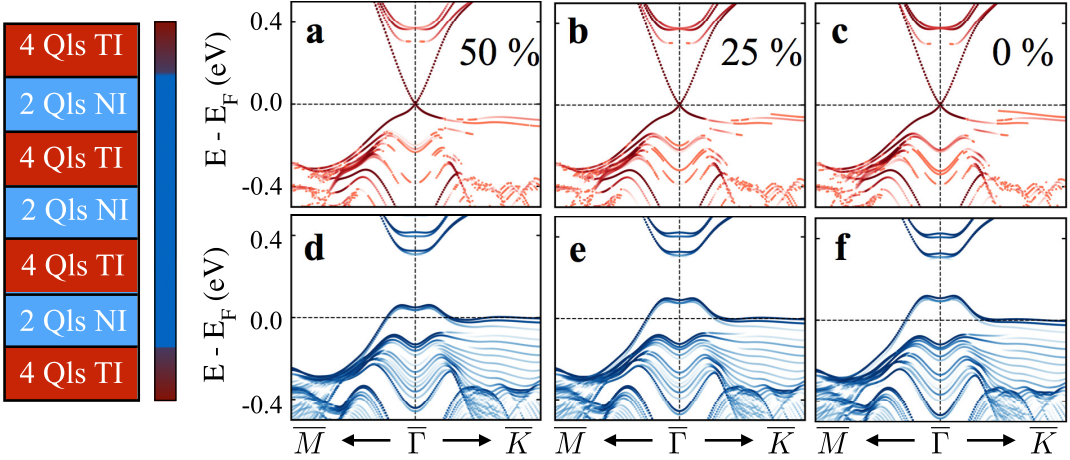

Figure 7. Band structure for a topological/normal insulator (TI/NI) finite heterostructure. The left panel shows the heterostructure schematic representation with four TI Qls (red) and two NI Qls (blue). In the NI region, the Bi SOC strength is varied from $50 \%$ of its full value to 0 . The eigenvalues spatial projection onto the outermost four TI Qls, at the surface, is shown in (a) 50\%, (b) 25\%, and (c) $0 \%$ of the full Bi SOC. In (d), (e), and (f), the projection is onto the middle region (TI and NI) of the slab. Dark red (dark blue) represents high (low) spectral weight at the projected region; see the colorbar.

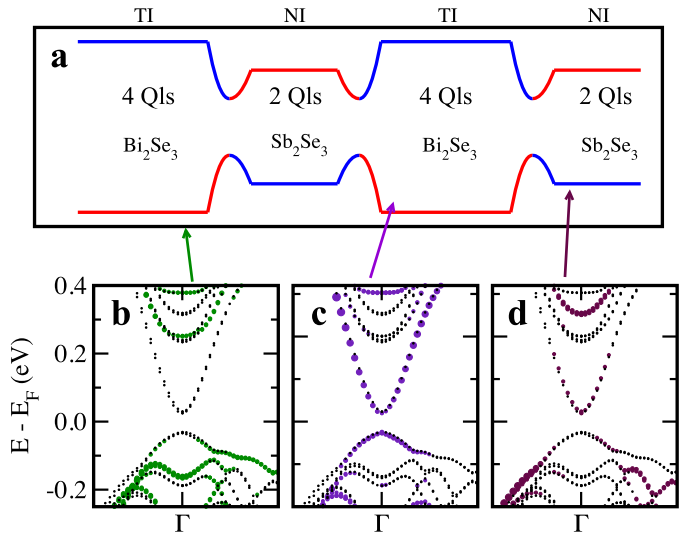

Figure 8. (a) TI/NI periodic superlattice schematic band structure. (b), (c), and (d) show the spatial eigenvalue projections of a one-Ql from the TI center region, TI, and NI at the interface, respectively.

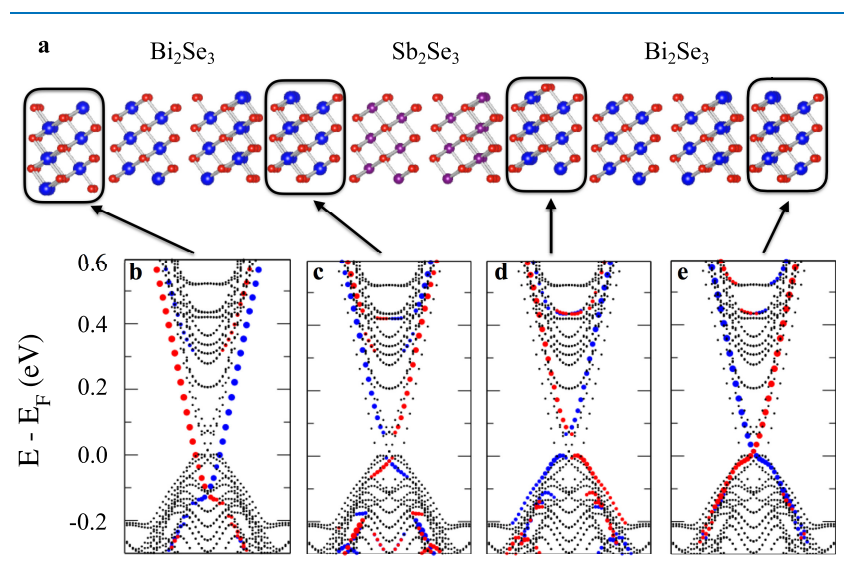

Figure 9. (a) 4/2/4 (TI/NI/TI) slab structure. (b)-(e) show spin projections on 1st, 4th, 7th, and 10th Qls, respectively. Blue and red are projections of opposite in-plane spin textures. The Fermi level is at zero energy.

$\mathrm{Bi}_{2} \mathrm{Se}_{3}$ symmetry since the unit cell demands three Qls, similar to stacking faults close to the surface. ${ }^{45}$

\section{CONCLUSIONS}

In conclusion, our calculations determine the topological state properties in heterostructures composed of TI/NI materials. Depending on the TI/NI heterostructure composition, topological states at adjacent interfaces hybridize, through the intervening NI material, opening a band gap at the Dirac crossing. We show that for NI layers thicker than the critical value $\left(L_{C}\right)$, the hybridization is suppressed and the bulk TPDS are restored. The topological states are spatially localized at the $\mathrm{TI} / \mathrm{NI}$ interface with a significant penetration in the NI region because of the proximity effect. Our calculations also indicate an inverse relationship between $L_{\mathrm{C}}$ and the NI gap $\left(\Delta_{\mathrm{NI}}\right)$, demonstrating that one should use a large-gap trivial material to obtain interface metallic Dirac states for thin Ni layers; otherwise, massive gaped topological states will be settled at the interfaces. Moreover, we also verified that TI and NI band alignment plays an important role in the stability of the TPDS. Even though the topological interface states are massive, they preserve mostly the in-plane spin texture. We have demonstrated that the gap of TI/NI heterostructure (or superlattice) can be fine-tuned by controlling the NI region chemical composition (which we mimic by varying the SOC strength) or its thickness. With these results, we expect to contribute to the understanding of the TI/NI heterostructure, which is important for the construction of devices using topological insulator materials.

\section{ASSOCIATED CONTENT}

\section{Supporting Information}

The Supporting Information is available free of charge on the ACS Publications website at DOI: 10.1021/acsomega.8b01836.

Bulk TB Hamiltonian along with the $\mathrm{Bi}_{2} \mathrm{Se}_{3}$ crystal unit cell (TXT)

PAOFLOW generation (TXT)

\section{AUTHOR INFORMATION}

\section{Corresponding Authors}

*E-mail: marcio.costa@lnnano.cnpem.br (M.C.).

*E-mail: adalberto.fazzio@Innano.cnpem.br (A.F.).

ORCID $\odot$

Marcio Costa: 0000-0003-1029-8202

Antônio T. Costa: 0000-0003-2899-6287

Tome M. Schmidt: 0000-0002-9121-8157

Notes

The authors declare no competing financial interest. 


\section{ACKNOWLEDGMENTS}

This work was supported by the Brazilian agencies FAPESP/ TEMÁTICO and CNPQ. We would like to acknowledge computing time provided by Laboratório de Computação Cientifica Avancada (Universidade de São Paulo). M.B.N. acknowledges support by DOD-ONR (N00014-13-1-0635, N00014-11-1-0136, and N00014-15-1-2863), the High-Performance Computing Center at the University of North Texas, and the Texas Advanced Computing Center at the University of Texas, Austin. We would also like to thank Prof. Yves Petroff and Dr. Ricardo Reis for the fruitful discussion regarding ARPES measurements.

\section{REFERENCES}

(1) Esaki, L.; Tsu, R. Superlattice and negative differential conductivity in semiconductors. IBM J. Res. Dev. 1970, 14, 61.

(2) Quantum Semiconductor Structures; Claude, W.; Borge, V., Eds.; Academic Press: San Diego, 1991.

(3) Baibich, M. N.; Broto, J. M.; Fert, A.; Van Dau, F. N.; Petroff, F.; Etienne, P.; Creuzet, G.; Friederich, A.; Chazelas, J. Giant Magnetoresistance of $(001) \mathrm{Fe} /(001) \mathrm{Cr}$ Magnetic Superlattices. Phys. Rev. Lett. 1988, 61, 2472-2475.

(4) Triscone, J.; Fischer, O.; Brunner, O.; Antognazza, L.; Kent, A. D.; Karkut, M. G. $\mathrm{YBa}_{2} \mathrm{Cu}_{3} \mathrm{O}_{7} / \mathrm{PrBa}_{2} \mathrm{Cu}_{3} \mathrm{O}_{7}$ Superlattices: Properties of Ultrathin Superconducting. Layers. Separated by Insulating Layers. Phys. Rev. Lett. 1990, 64, 804-807.

(5) Kane, C. L.; Mele, E. J. $Z_{2}$ Topological Order and the Quantum Spin Hall Effect. Phys. Rev. Lett. 2005, 95, No. 146802.

(6) Bernevig, B. A.; Hughes, T. L.; Zhang, S.-C. Quantum Spin Hall Effect and Topological Phase Transition in HgTe Quantum Wells. Science 2006, 314, 1757-1761.

(7) Fu, L.; Kane, C. L.; Mele, E. J. Topological Insulators in Three Dimensions. Phys. Rev. Lett. 2007, 98, No. 106803.

(8) Belopolski, I.; Xu, S.-Y.; Koirala, N.; Liu, C.; Bian, G.; Strocov, V. N.; Chang, G.; Neupane, M.; Alidoust, N.; Sanchez, D.; Zheng, H.; Brahlek, M.; Rogalev, V.; Kim, T.; Plumb, N. C.; Chen, C.; Bertran, F.; le Fèvre, P.; Taleb-Ibrahimi, A.; Asensio, M.-C.; Shi, M.; Lin, H.; Hoesch, M.; Oh, S.; Hasan, M. Z. A novel artificial condensed matter lattice and a new platform for one-dimensional topological phases. Sci. Adv. 2017, 3, No. e1501692.

(9) Krizman, G.; Assaf, B. A.; Phuphachong, T.; Bauer, G.; Springholz, G.; Bastard, G.; Ferreira, R.; de Vaulchier, L. A.; Guldner, Y. Tunable Dirac interface states in topological superlattices. Phys. Rev. B 2018, 98, No. 075303.

(10) Hirahara, T.; Bihlmayer, G.; Sakamoto, Y.; Yamada, M.; Miyazaki, H.; Kimura, S.-i.; Blügel, S.; Hasegawa, S. Interfacing 2D and 3D Topological Insulators: $\mathrm{Bi}(111)$ Bilayer on $\mathrm{Bi}_{2} \mathrm{Te}_{3}$. Phys. Rev. Lett. 2011, 107, No. 166801.

(11) Berntsen, M. H.; Götberg, O.; Wojek, B. M.; Tjernberg, O. Direct observation of decoupled Dirac states at the interface between topological and normal insulators. Phys. Rev. B 2013, 88, No. 195132.

(12) Lee, H.; Yazyev, O. V. Lattice-matched heterojunctions between topological and normal insulators: A first-principles study. Phys. Rev. B 2017, 95, No. 085304.

(13) Takahashi, R.; Murakami, S. Gapless Interface States between Topological Insulators with Opposite Dirac Velocities. Phys. Rev. Lett. 2011, 107, No. 166805.

(14) Aramberri, H.; Muñoz, M. C. Strain effects in topological insulators: Topological order and the emergence of switchable topological interface states in $\mathbf{S b}_{2} \mathbf{T e}_{3} / \mathbf{B i}_{2} \mathbf{T e} \mathbf{e}_{3}$ heterojunctions. Phys. Rev. B 2017, 95, No. 205422.

(15) Seixas, L.; West, D.; Fazzio, A.; Zhang, S. B. Vertical twinning of the Dirac cone at the interface between topological insulators and semiconductors. Nat. Commun. 2015, 6, No. 7630.

(16) de Oliveira, I. S.; Miwa, R. H. Pyridine intercalated Bi 2 Se 3 heterostructures: controlling the topologically protected states. Nanotechnology 2016, 27, No. 035704.
(17) Kim, J.; Kim, K.-W.; Wang, H.; Sinova, J.; Wu, R. Understanding the Giant Enhancement of Exchange Interaction in $\mathrm{Bi}_{2} \mathrm{Se}_{3}-\mathrm{EuS}$ Heterostructures. Phys. Rev. Lett. 2017, 119, No. 027201.

(18) Freitas, W. A.; Fazzio, A.; Schmidt, T. M. Topological states of nanoscale Bi2Se3 interfaced with AlN. Appl. Phys. Lett. 2016, 109, No. 131601.

(19) Hutasoit, J. A.; Stanescu, T. D. Induced spin texture in semiconductor/topological insulator heterostructures. Phys. Rev. B 2011, 84, No. 085103.

(20) Zhang, J.; Triola, C.; Rossi, E. Proximity Effect in GrapheneTopological-Insulator Heterostructures. Phys. Rev. Lett. 2014, 112, No. 096802.

(21) Usanmaz, D.; Nath, P.; Toher, C.; Plata, J. J.; Friedrich, R.; Fornari, M.; Buongiorno Nardelli, M.; Curtarolo, S. Spinodal superlattices of topological insulators. Chem. Mater. 2018, 30, 2331-2340.

(22) Shoman, T.; Takayama, A.; Sato, T.; Souma, S.; Takahashi, T.; Oguchi, T.; Segawa, K.; Ando, Y. Topological proximity effect in a topological insulator hybrid. Nat. Commun. 2015, 6, No. 6547.

(23) Agapito, L. A.; Ferretti, A.; Calzolari, A.; Curtarolo, S.; Buongiorno Nardelli, M. Effective and accurate representation of extended Bloch states on finite Hilbert spaces. Phys. Rev. B 2013, 88, No. 165127.

(24) Agapito, L. A.; Curtarolo, S.; Buongiorno Nardelli, M. Reformulation of DFT $+U$ as a Pseudohybrid Hubbard Density Functional for Accelerated Materials Discovery. Phys. Rev. X 2015, 5, No. 011006.

(25) Agapito, L. A.; Fornari, M.; Ceresoli, D.; Ferretti, A.; Curtarolo, S.; Nardelli, M. B. Accurate tight-binding Hamiltonians for twodimensional and layered materials. Phys. Rev. B 2016, 93, No. 125137.

(26) Agapito, L. A.; Ismail-Beigi, S.; Curtarolo, S.; Fornari, M.; Nardelli, M. B. Accurate tight-binding Hamiltonian matrices from $a b$ initio calculations: Minimal basis sets. Phys. Rev. B 2016, 93, No. 035104.

(27) Nardelli, M. B.; Cerasoli, F. T.; Costa, M.; Curtarolo, S.; Gennaro, R. D.; Fornari, M.; Liyanage, L.; Supka, A. R.; Wang, H. PAOFLOW: A utility to construct and operate on ab initio Hamiltonians from the projections of electronic wavefunctions on atomic orbital bases, including characterization of topological materials. Comput. Mater. Sci. 2018, 143, 462-472.

(28) Corso, A. D.; Conte, A. M. Spin-orbit coupling with ultrasoft pseudopotentials: Application to Au and Pt. Phys. Rev. B 2005, 71, No. 115106.

(29) Abate, E.; Asdente, M. Tight-Binding Calculation of $3 d$ Bands of Fe with and without Spin-Orbit Coupling. Phys. Rev. 1965, 140, A1303-A1308.

(30) Zhang, H.; Liu, C.-X.; Qi, X.-L.; Dai, X.; Fang, Z.; Zhang, S.-C. Topological insulators in $\mathrm{Bi} 2 \mathrm{Se} 3, \mathrm{Bi} 2 \mathrm{Te} 3$ and $\mathrm{Sb} 2 \mathrm{Te} 3$ with a single Dirac cone on the surface. Nat. Phys. 2009, 5, 438-442.

(31) Hohenberg, P.; Kohn, W. Inhomogeneous Electron Gas. Phys. Rev. 1964, 136, B864-B871.

(32) Kohn, W.; Sham, L. J. Self-Consistent Equations Including Exchange and Correlation Effects. Phys. Rev. 1965, 140, A1133A1138.

(33) Giannozzi, P.; Baroni, S.; Bonini, N.; Calandra, M.; Car, R.; Cavazzoni, C.; Ceresoli, D.; Chiarotti, G. L.; Cococcioni, M.; Dabo, I.; Dal Corso, A.; de Gironcoli, S.; Fabris, S.; Fratesi, G.; Gebauer, R.; Gerstmann, U.; Gougoussis, C.; Kokalj, A.; Lazzeri, M.; MartinSamos, L.; Marzari, N.; Mauri, F.; Mazzarello, R.; Paolini, S.; Pasquarello, A.; Paulatto, L.; Sbraccia, C.; Scandolo, S.; Sclauzero, G.; Seitsonen, A. P.; Smogunov, A.; Umari, P.; Wentzcovitch, R. M. UANTUM ESPRESSO: a modular and open-source software project for quantum simulations of materials. J. Phys.: Condens. Matter 2009, 21, No. 395502.

(34) Giannozzi, P.; Andreussi, O.; Brumme, T.; Bunau, O.; Nardelli, M. B.; Calandra, M.; Car, R.; Cavazzoni, C.; Ceresoli, D.; Cococcioni, M.; Colonna, N.; Carnimeo, I.; Corso, A. D.; de Gironcoli, S.; Delugas, P.; DiStasio, R. A., Jr.; Ferretti, A.; Floris, A.; Fratesi, G.; Fugallo, G.; Gebauer, R.; Gerstmann, U.; Giustino, F.; Gorni, T.; Jia, 
J.; Kawamura, M.; Ko, H.-Y.; Kokalj, A.; Küçükbenli, E.; Lazzeri, M.; Marsili, M.; Marzari, N.; Mauri, F.; Nguyen, N. L.; Nguyen, H.-V.; dela Roza, A. O.; Paulatto, L.; Poncé, S.; Rocca, D.; Sabatini, R.; Santra, B.; Schlipf, M.; Seitsonen, A. P.; Smogunov, A.; Timrov, I.; Thonhauser, T.; Umari, P.; Vast, N.; Wu, X.; Baroni, S. Advanced capabilities for materials modelling with Quantum ESPRESSO. J. Phys.: Condens. Matter 2017, 29, No. 465901.

(35) Kresse, G.; Furthmüller, J. Efficiency of ab-initio total energy calculations for metals and semiconductors using a plane-wave basis set. Comput. Mater. Sci. 1996, 6, 15-50.

(36) Kresse, G.; Furthmuller, J. Efficient iterative schemes for ab initio total-energy calculations using a plane-wave basis set. Phys. Rev. B 1996, 54, 11169-11186.

(37) Perdew, J. P.; Burke, K.; Ernzerhof, M. Generalized Gradient Approximation Made Simple. Phys. Rev. Lett. 1996, 77, 3865-3868.

(38) Kresse, G.; Joubert, D. From ultrasoft pseudopotentials to the projector augmented-wave method. Phys. Rev. B 1999, 59, 17581775

(39) Nakayama, K.; Eto, K.; Tanaka, Y.; Sato, T.; Souma, S.; Takahashi, T.; Segawa, K.; Ando, Y. Manipulation of Topological States and the Bulk Band Gap Using Natural Heterostructures of a Topological Insulator. Phys. Rev. Lett. 2012, 109, No. 236804.

(40) Jin, K.-H.; Yeom, H. W.; Jhi, S.-H. Band structure engineering of topological insulator heterojunctions. Phys. Rev. B 2016, 93, No. 075308

(41) Yazyev, O. V.; Moore, J. E.; Louie, S. G. Spin Polarization and Transport of Surface States in the Topological Insulators $\mathrm{Bi}_{2} \mathrm{Se}_{3}$ and $\mathrm{Bi}_{2} \mathrm{Te}_{3}$ from First Principles. Phys. Rev. Lett. 2010, 105, No. 266806.

(42) Acosta, C. M.; Lima, M. P.; da Silva, A. J. R.; Fazzio, A.; Lewenkopf, C. H. Tight-binding model for the band dispersion in rhombohedral topological insulators over the whole Brillouin zone. Phys. Rev. B 2018, 98, No. 035106.

(43) Abdalla, L. B.; Jose, E. P.; Schmidt, T. M.; Miwa, R. H.; Fazzio, A. Topological phase transitions of (Bix Sb1-x ) 2 Se 3 alloys by density functional theory. J. Phys.: Condens. Matter 2015, 27, No. 255501.

(44) Shan, W.-Y.; Lu, H.-Z.; Shen, S.-Q. Effective continuous model for surface states and thin films of three-dimensional topological insulators. New J. Phys. 2010, 12, No. 043048.

(45) Abdalla, L. B.; Padilha, J. E.; Schmidt, T. M.; Miwa, R. H.; Fazzio, A. Topological phase transitions of $\operatorname{BixSb}(1-\mathrm{x}) 2 \mathrm{Se} 3$ alloys by density functional theory. J. Phys.: Condens. Matter 2015, 27, No. 255501. 